\title{
Comparing Outcomes of HIV versus Non-HIV Patients Requiring Mechanical Ventilation
}

\author{
Vikas Pathak, MD; lliana Samara Hurtado Rendon, MD; Shebli Atrash, MD; Vinay Prasad Rao Gagadam, MD; \\ Kaushik Bhunia, MD; Syam Prasad Mallampalli, MD; Vijay Vegesna, MD; Mahesh Mani Dangal, MD; and \\ Ronald L. Ciubotaru, MD
}

\begin{abstract}
Background: Mechanical ventilation (MV) is a predictor of mortality in patients infected with human immunodeficiency virus (HIV) in the intensive care unit (ICU). Patients with HIV-infections are admitted to the ICU for a variety of reasons that frequently require intubation. While survival rates for HIV-infected patients continue to improve, ICU admission rates have remained consistent.
\end{abstract}

Methods: To observe the consequences of MV in HIV-infected patients, we conducted a retrospective chart review on patients with HIV $(n=55)$ vs. matched HIV-negative patients $(n=55)$ who required MV over a one-year period and compared the groups for differences in outcome and complications.

Results: The HIV group had twice the number of deaths ( $44 \%$ vs. $22 \%$, all-cause mortality) $(P=0.0 \mathrm{I})$. Among the HIV-positive group, 5 of 55 patients required tracheostomy and prolonged MV, compared to 15 of 55 in the control group ( $9 \%$ and $27 \%$, respectively). Successful extubation was virtually identical ( $47 \%$ MV vs. $50 \%$ control). Ventilator-associated pneumonia (VAP) was significantly higher among HIV-positive cases ( 39 of 55 HIV vs. 14 of 55 non-HIV) $(P=0.05)$. Regression analysis revealed that hypotension, hypoalbuminemia, and fever predicted a poorer outcome. Low CD4 cell counts were strongly associated with mortality.

Conclusion: HIV-infected patients requiring MV have significantly higher mortality and VAP rates than HIV-negative patients. Since VAP is associated with a poor prognosis, discovering ways to prevent it in the HIV-infected patient may improve outcome.

Keywords: Intensive Care Unit (ICU); HIV; Mechanical ventilation; Ventilator-associated pneumonia

$\mathrm{H}$ are admitted to the intensive care unit (ICU) for a variety of indications that frequently require intubation. Though the survival rates of HIV-infected patients continue to improve in the highly active antiretroviral therapy (HAART) era, ${ }^{1}$ the severity of the acute illnesses requiring ICU admission has remained unchanged over time. ${ }^{2}$ Due to longer survival, the annual rate of ICU admissions for HIV-positive patients has remained the same, despite improved treatment approaches and increased understanding of the pathology underlying the disease. ${ }^{1,2}$ Mechanical ventilation (MV) has been cited as one of the predictors of mortality in HIV-infected patients

Corresponding Author: Vikas Pathak MD; Pulmonary Disease and Critical Care Medicine; University of North Carolina School of Medicine; I30 Mason Farm Rd; Chapel Hill, NC 27599 USA; Tel: (919) 966-2531; Fax: (919) 966-70I3; E-mail: drvikaspathak@gmail.com requiring ICU support; ${ }^{1,3}$ however, these studies lacked a control group. We directly compared the outcomes of MV in HIV-infected patients versus HIV-negative patients using a case-control design to compare length of ventilation and rates of mortality among these two demographics. This study tested the hypothesis that mechanical ventilation in HIVpositive patients predicts increased ICU mortality and complications when compared to HIV-negative patients. We identified a group of HIV-negative patients to serve as a control group in order to have a comparable reference population for evaluation of outcomes in our index population of HIV-infected patients.

Received: December 17, 2010

Ist Revision: May II, 201I

2nd Revision: September 23, 2011

Accepted: September 28, $201 \mathrm{I}$ 
Table 1. Baseline characteristics of HIV and Non-HIV patients admitted to the ICU*

\begin{tabular}{|c|c|c|}
\hline Characteristics & $\begin{array}{l}\text { Cases (HIV+) } \\
\text { n (\%) }\end{array}$ & $\begin{array}{l}\text { Controls (HIV-) } \\
\text { n (\%) }\end{array}$ \\
\hline \multicolumn{3}{|l|}{ Gender } \\
\hline Male & $30(55 \%)$ & $32(58 \%)$ \\
\hline Female & $25(45 \%)$ & $23(42 \%)$ \\
\hline Age (years) - Mean (range) & $50(33-75)$ & $52(32-78)$ \\
\hline \multicolumn{3}{|l|}{ Race } \\
\hline White & $5(9 \%)$ & $8(15 \%)$ \\
\hline Black & $25(45 \%)$ & $23(42 \%)$ \\
\hline Hispanic & $20(36 \%)$ & $18(33 \%)$ \\
\hline Asian & $5(9 \%)$ & $6(11 \%)$ \\
\hline PCP Prophylaxis & $5(9 \%)$ & $\mathrm{N} / \mathrm{A}$ \\
\hline HAART & $8(15 \%)$ & $\mathrm{N} / \mathrm{A}$ \\
\hline CD4 cell count -Median (range) & $102(0-508)$ & $\mathrm{N} / \mathrm{A}$ \\
\hline AIDS & $47(85 \%)$ & $\mathrm{N} / \mathrm{A}$ \\
\hline APACHE II - Median (range) & $18(12-34)$ & $18(10-30)$ \\
\hline \multicolumn{3}{|c|}{ Relative incidence of potential complications } \\
\hline VAP & $39(71 \%)$ & $14(25 \%)$ \\
\hline Spontaneous pneumonia & $1(2 \%)$ & $0(0 \%)$ \\
\hline DVT & $1(2 \%)$ & $3(5 \%)$ \\
\hline Infections** & $19(35 \%)$ & $15(27 \%)$ \\
\hline
\end{tabular}

${ }^{\star} \mathrm{N}=55$ patients per group; ${ }^{* *}$ including line sepsis, urinary tract infection, and bacteremia

APACHE, Acute Physiology and Chronic Health Evaluation; VAP, ventilator-associated pneumonia; DVT, deep vein thrombosis; PCP, Pneumocystis jiroveci pneumonia; HAART, highly active antiretroviral therapy

\section{Materials and Methods}

Study Design and Subjects

A retrospective, case-control study was undertaken in a cohort of all HIV-infected adults who required MV and had been admitted to the ICU at New York's St. Barnabas Hospital from January 1, 2009 through December 31, 2009. St. Barnabas Hospital is an inner city hospital with 450 beds and 32 mixed medical/surgical ICU beds. After approval by the institutional review board, a computerized search of St. Barnabas Hospital ICU admissions using the International Classification of Diseases, ninth revision (ICD 9), diagnostic code for HIV (042) identified all patients with HIV who had been admitted to the ICU during our defined time period. Out of a total of $150 \mathrm{HIV}$-infected patients admitted to the ICU, 55 had been intubated, and these were all included in the study as cases.

Investigators reviewed the medical records of patients using a standardized data collection tool in order to standardize data collection across investigators. The charts of all adults admitted during the study were abstracted, and the control group of HIV-negative patients was matched as closely as possible to HIV cases for similar demographic data including age, sex, race, admission diagnosis, reason for intubation and Acute Physiology and Chronic Health Evaluation (APACHE) II score (table 1). Due to lack of information regarding current medications and a general lack of compliance with HAART in our patient population, patients did not receive anti-retroviral treatment during ICU stay to avoid potential interaction with other treatments during acute index episode of tracheostomy or ventilator-associated pneumonia (VAP).
Patients enrolled as controls were required to have had a negative HIV test within one month of screening.

Outcomes of interest included the following: death in the ICU, time to tracheostomy, proportion requiring tracheotomy, and complications of mechanical ventilation. A primary endpoint was the rate of VAP among HIV-positive patients. At St. Barnabas Hospital the definition of VAP was fever, elevated white blood cells, purulent secretions, and new infiltrate on chest radiograph. Diagnosis of VAP was assigned based on the use of a data collection tool to identify the presence of above-mentioned criteria and was validated by the lead author through manual chart abstraction.

\section{Data Collection}

Data were collected from 55 HIV-infected patients requiring MV (cases) and 55 HIV-negative patients requiring MV (controls). Four investigators reviewed the medical records of patients using a standardized data collection tool that captured demographic data (age, sex, race), ICU admission diagnoses, indication for MV, ICU course and length of stay (equivalent to length of intubation), complications during ICU stay, and death. Quality control was done by two additional investigators who cross-checked all the collected data. Patients were scored on one of three possible outcomes: (1) weaning, as defined by successful extubation for more than 24 hours and transfer to a general medical floor; (2) tracheostomy within 10 to 21 days after intubation followed by patient transfer to the ventilator step-down unit or nursing home with ventilator facility; or (3) death. ICU stay was defined as time until transfer or death, as the total length of hospitalization or 
Table 2. Principle Outcomes.

\begin{tabular}{lllll}
\hline Outcome & HIV+ & HIV- & Total & P value \\
\hline Weaned & n (\%) & n (\%) & n (\%) & 0.08 \\
Tracheostomy & $26(47 \%)$ & $28(51 \%)$ & $54(49 \%)$ & 0.01 \\
Death & $5(9 \%)$ & $15(27 \%)$ & $20(18 \%)$ & 0.01 \\
\hline
\end{tabular}

institutionalization could not be calculated since some patients were lost to follow-up or remained chronically on ventilators. For the purposes of this study, length of intubation was considered equivalent to length of ICU stay. Patients requiring tracheostomy were considered to have prolonged ventilation. Other clinical information collected included cluster difference 4 (CD4) cell count, serum albumin level, and chest radiograph findings. Data related to microbiological/pathological analysis of body fluids including sputum and tracheal aspirates were also captured. The APACHE II scores were calculated for both groups to compare severity of illness. If patients were readmitted to the ICU during the same hospitalization, only the data from the first admission were recorded.

\section{Statistical Analysis}

Continuous data were compared using the Student's $t$ test and comparisons of frequencies were made with the Chi-square test. Student's $t$ test was done to compare APACHE II scores. We used univariate followed by multivariate logistic regression to test for differences among two or more independent groups. Statistical Package for the Social Sciences (SPSS, version 13.0) was used for statistical analysis. Statistical significance was predetermined in reference to a $P$ value of $<0.05$.

\section{Results}

A total of 110 patients meeting inclusion criteria were evaluated in this study. Case subjects consisted of 55 patients who were HIV-positive and needed MV, and controls were represented by 55 patients who were HIV-negative and needed MV. Patient baseline characteristics in areas of demographics, clinical factors, and level of illness were evaluated for both cases and controls (table 1). Due to systematic matching, data were not analyzed on parameters including age, sex, race, admission diagnosis, or reason for intubation among the two groups. APACHE II scores ranged from 10 to 34 with a mean of 18 in both case and control groups.

Principle patient outcomes of this study were (1) weaning from mechanical ventilation, (2) tracheostomy, or (3) death (table 2). Of patients who were weaned from the ventilator, 26 (47\%) were HIV-positive cases and 28 (51\%) were HIVnegative controls (table 2). The average weaning time, defined as successful extubation for 24 hours or more, in HIV-positive patients was on day 6 (range 1-10 days), versus day 7 (range 1-12 days) in HIV-negative patients. Significantly fewer HIV-positive than HIV-negative patients required tracheostomy. Of the $55 \mathrm{HIV}$-positive patients, $5(9 \%)$ had a tracheostomy, while 15 of the 55 patients $(27 \%)$ in the HIVnegative group had a tracheostomy $(P=0.01)$ (table 2). Tracheostomy was performed between day 10 and day 21 of MV following patient failure of the spontaneous breathing trial and multiple weaning attempts while in the ICU. The rate of tracheostomy among the control group was 3 times higher than among the HIV-positive group. Patients requiring tracheostomy were considered to have prolonged ventilation. The average number of days to tracheostomy among patients with HIV was 12 (range 10-21 days) and 14 days (range 10-21 days) among HIV-negative controls. The rate of weaning or days to tracheostomy between groups was not statistically significant. There was, however, considerable difference in mortality rates among the HIV-positive cases $(44 \% ; 24 / 55)$ versus the control group $(22 \% ; 12 / 55)$ $(P=0.01)$.

Table 3. Analysis of the tracheal aspirate organisms isolated in patients with ventilator-associated pneumonia.

\begin{tabular}{lll}
\hline Organism & Cases (HIV+) & Controls (HIV-) \\
\hline Acinetobacter baumannii & $4(10 \%)$ & $5(36 \%)$ \\
Escherichia coli & $0(0 \%)$ & $3(21 \%)$ \\
Klebsiella pneumoniae & $7(18 \%)$ & $3(21 \%)$ \\
Mycobacterium avium-intracellulare & $3(8 \%)$ & $0(0 \%)$ \\
Pseudomonas aeruginosa & $11(28 \%)$ & $3(21 \%)$ \\
Staphylococcus aureus & & $0(0 \%)$ \\
$\quad$ Methicillin-resistant & $3(8 \%)$ & $0(0 \%)$ \\
Mroup A Streptococcus & $4(10 \%)$ & $0(0 \%)$ \\
Total & $2(5 \%)$ & $14(100 \%)$ \\
\hline
\end{tabular}


Table 4. CD4 count and association with ventilator-associated pneumonia (VAP).

\begin{tabular}{lll}
\hline $\begin{array}{l}\text { CD4 Count, } \\
\text { cells } / \mathbf{m m}^{3}\end{array}$ & $\begin{array}{l}\text { Patients, } \\
\mathbf{n}\end{array}$ & $\begin{array}{l}\text { Incidence of VAP, } \\
\mathbf{n}(\%)\end{array}$ \\
\hline $0-49$ & 21 & $17(80 \%)$ \\
$50-99$ & 7 & $5(71 \%)$ \\
$100-199$ & 12 & $7(58 \%)$ \\
$\geq 200$ & 11 & $9(81 \%)$ \\
Unknown & 5 & $1(25 \%)$ \\
Total & 55 & $39(71 \%)$ \\
\hline
\end{tabular}

The most frequent complication of MV noted in this study among patients with HIV was VAP. Among HIV-infected patients, 39 of 55 patients (71\%) had VAP, compared to 14 of 55 patients $(25 \%)$ in the non-HIV group $(P<0.001)$ (table 1$)$. All five patients in the HIV-positive group who underwent tracheostomy developed VAP, whereas only 6 of $15(40 \%)$ patients who underwent tracheostomy in the non-HIV group developed VAP $(P=0.02)$.

Organisms most frequently isolated from patients with VAP in the HIV-positive group included Pseudomonas aeruginosa (28\%), Klebsiella pneumoniae (18\%), and Staphylococcus aureus (18\%). The most frequent bacterial isolate among the HIV-negative patients who developed VAP was Acinetobacter baumannii (36\%). A complete list of infectious agents encountered in the context of VAP is shown in table 3.

CD4 cell counts of less than 200 cells $/ \mathrm{mm}^{3}$ were measured in 29 of $40(72 \%)$ of HIV-positive patients who developed VAP (table 4). Surprisingly, this was similar to the rate of VAP in patients with CD4 cell counts of $\geq 200$ cells $/ \mathrm{mm}^{3}$ where 9 of $11(81 \%)$ developed VAP (table 4$)$, suggesting that patients with low CD4 cell counts are not at increased risk for VAP as might be expected. Besides VAP, there was no significant difference between the HIV-positive patients and the control group with respect to other complications (spontaneous pneumonia, DVT, and infections) listed in table 1.

Admission for pneumonia was significantly associated with mortality in the HIV-positive group $(P<0.02)$ (table 5). The main indications for intubation in both groups included (1) respiratory failure (defined as tachypnea with clinically apparent respiratory distress, $\mathrm{PaO}_{2}<60$ and/or $\mathrm{Pco}_{2}>50$ and hypoxemia requiring MV delivered by endotracheal tube), followed by (2) airway protection, and (3) sepsis (data not shown). Prolonged MV rates, as assessed by the need for tracheostomy, were three times higher in HIV-negative patients compared to HIV-positive patients (table 2). Univariate followed by multivariate logistic regression analysis showed that low serum albumin $(<21 \mathrm{~g} / \mathrm{L})(P<0.001)$, elevated temperature $(P=0.03)$, low systolic blood pressure $(<85 \mathrm{mmHg})(P=0.01)$, and VAP $(P=0.05)$ were the strongest predictors of mortality in HIV-infected patients (table 6).

\section{Intergroup Comparison Among Cases}

Based on the Centers for Disease Control 1993 definition, $85 \%$ of the HIV-positive patients had acquired immunodeficiency syndrome (AIDS). ${ }^{4}$ It is noteworthy that among the HIV-positive cases, all 24 patients who died had AIDS. A total of 31 patients $(56 \%)$ in the HIV-positive group survived the ICU stay, of which 23 (74\%) had AIDS. HIV-positive patients with AIDS were significantly less likely to survive than those without AIDS $(P<0.01$, Chi-squared test). Of the 24 HIV-positive patients who died, 16 were males and 8 were

Table 5. Admission diagnoses and mortality.

\begin{tabular}{|c|c|c|c|c|c|}
\hline \multirow[b]{2}{*}{ Diagnosis } & \multicolumn{2}{|c|}{ Cases (HIV+) } & \multicolumn{2}{|c|}{ Controls (HIV-) } & \multirow[b]{2}{*}{$P$ value* } \\
\hline & $\mathbf{n}$ & $\begin{array}{c}\text { Survival, } \\
\text { n (\%) }\end{array}$ & $\mathbf{n}$ & $\begin{array}{c}\text { Survival, } \\
\text { n (\%) }\end{array}$ & \\
\hline Pneumonia** & 10 & $3(30 \%)$ & 8 & $7(87 \%)$ & $<0.02$ \\
\hline Asthma & 5 & $5(100 \%)$ & 8 & $7(87 \%)$ & ns \\
\hline COPD & 5 & $5(100 \%)$ & 7 & $6(86 \%)$ & ns \\
\hline CHF/pulmonary edema & 6 & $5(83 \%)$ & 5 & $2(40 \%)$ & ns \\
\hline Sepsis & 9 & $3(33 \%)$ & 7 & $5(71 \%)$ & ns \\
\hline Cardiac arrest & 5 & $3(60 \%)$ & 4 & $3(75 \%)$ & ns \\
\hline Change in mental status & 10 & $4(40 \%)$ & 8 & $6(75 \%)$ & ns \\
\hline Seizure & 3 & $3(100 \%)$ & 5 & $5(100 \%)$ & ns \\
\hline Other & 2 & $0(0 \%)$ & 3 & $2(66 \%)$ & ns \\
\hline Total & 55 & $31(56 \%)$ & 55 & $43(78 \%)$ & $<0.02$ \\
\hline
\end{tabular}

*survival difference between HIV+ and HIV- patients, Chi-square test; ns, not significant

${ }^{\star *}$ No cases of pneumonia were found to be caused by Pneumocystis jiroveci

COPD, chronic obstructive pulmonary disease; $\mathrm{CHF}$, congestive heart failure 
Table 6. Multivariate analysis of predictors associated with increased mortality.

\begin{tabular}{|c|c|c|c|c|}
\hline \multicolumn{5}{|c|}{ Case Group (HIV+) } \\
\hline Predictor & $\begin{array}{l}\text { Survivors } \\
(n=24)\end{array}$ & $\begin{array}{l}\text { Non-Survivors } \\
(n=31)\end{array}$ & $P$ value & $\begin{array}{l}\text { Odds ratio } \\
(95 \% \mathrm{Cl})\end{array}$ \\
\hline Albumin $(<21 \mathrm{~g} / \mathrm{L})$, mean & $25 \mathrm{~g} / \mathrm{L}$ & $18 \mathrm{~g} / \mathrm{L}$ & $<0.001$ & $4.0(2.5-5.5)$ \\
\hline High temperature, mean & $98^{\circ} \mathrm{F}$ & $100.1^{\circ} \mathrm{F}$ & 0.03 & $3.1(1.8-6.0)$ \\
\hline Low Systolic BP (<85 mmHg), mean & 96 & 84 & 0.01 & $3.0(2.5-5.0)$ \\
\hline VAP, n & 14 & 10 & 0.03 & $2.8(1.5-3.1)$ \\
\hline
\end{tabular}

females. The mean age of the HIV-positive patients who died was 52 years, and the mean APACHE II score was 18, which was not statistically different from HIV-positive patients who survived the ICU stay. The mean age of patients who survived was 50 years (range 33 to 75 years). Among HIV-positive patients who survived the ICU stay, the mean CD4 cell count was 208 cells $/ \mathrm{mm}^{3}$ (range 20-508 cells $/ \mathrm{mm}^{3}$ ), while the mean CD4 cell count was 88 cells $/ \mathrm{mm}^{3}$ (range $0-350$ cells $/ \mathrm{mm}^{3}$ ) in the HIV-positive patients who died. The CD4 cell count showed negative correlation with time to weaning (correlation coefficient $=-0.497)$, suggesting that as the CD4 cell count increased, the number of days to weaning decreased $(P=0.02)$. Consistent with the association between low CD4 count and death, HIV-positive patients admitted for infectious causes, including pneumonia, sepsis, and change in mental status, were significantly less likely to survive (34\%) than those admitted for non-infectious causes, including asthma, chronic obstructive pulmonary disease (COPD), congestive heart failure $(\mathrm{CHF}) /$ pulmonary edema, seizures, and others $(86 \%)$ $(P<0.001)$.

\section{Discussion}

Acute respiratory failure is the most common reason for ICU admission and MV in HIV-infected patients, accounting for approximately $25 \%$ to $50 \%$ of ICU admissions. ${ }^{5-10}$ Before the availability of HAART, Pneumocystis jiroveci pneumonia (PCP) was the main cause of respiratory failure. ${ }^{6,8,11}$ Due to a better understanding of the disease, HAART, and the availability of prophylaxis for PCP, bacterial pneumonia has emerged as the main cause of respiratory failure and ICU admission. ${ }^{10,12-14}$ In the present study, respiratory failure was the most common indication for ICU intubation in HIVinfected patients, followed by airway protection and sepsis. Several studies suggest that mortality following $\mathrm{MV}$ is particularly high in HIV-infected patients.

De Palo et $\mathrm{al}^{3}$ reported a mortality rate of $79 \%$ (18 of 23 ) among HIV-infected patients who required MV compared to only $33 \%$ (4 of 12) that did not require MV. Nickas et $\mathrm{al}^{9}$ also reported adverse outcomes among HIV-infected patients who required MV with a mortality rate of 51\% (124 of 245) compared to those who did not require MV 18\% (36 of 196). However, to date no study has compared an HIV-positive population to a carefully selected group of HIV-negative controls. We directly compared outcomes in HIV-infected patients requiring $\mathrm{MV}$ to those of HIV-negative patients. In the present study, we found a significant increase in mortality in patients with HIV following MV (44\%) compared to patients without HIV $(22 \%)(P=0.01)$, confirming the increased risk for mortality associated with $\mathrm{MV}$ in HIVpositive patients in previous studies.

HIV-positive cases and HIV-negative controls were admitted for infectious causes at a similar rate, yet HIV-positive patients were significantly less likely to survive the ICU stay (34\%) if admitted for an infectious cause than a non-infectious cause $(81 \%)(P<0.001)$. In HIV-negative patients survival was similar whether admission diagnosis was infectious $(78 \%)$ or non-infectious $(78 \%)$. Whether decreased survival in HIV-positive patients is due to immunosuppression and an inability to mount an effective immune response in the face of infection or due to the type of infection to which these patients are susceptible is unknown. Additionally, following MV, HIV-positive patients were more likely to develop VAP (71\%) than HIV-negative patients (25\%) (table 1). In the present study, VAP was most frequently associated with $P$. aeruginosa, K. pneumoniae, and S. aureus infection in HIVpositive patients and A. baumanii in HIV-negative patients (table 3). The small number of patients infected with each organism does not allow for statistical analysis. Together, these data suggest that infection, both as admission diagnosis and as a complication following $\mathrm{MV}$, may play a role in the increased mortality observed in HIV-positive patients.

In the present study, we also performed intergroup comparisons between HIV-infected patients who died with those who survived the ICU stay. HIV-positive patients with AIDS were significantly less likely to survive following MV in the ICU than HIV-positive patients who had not yet progressed to AIDS. We also demonstrated that HIV-infected patients with AIDS who were admitted due to infectious causes had increased mortality compared to patients admitted for noninfectious reasons such as chronic obstructive pulmonary disease, asthma, and congestive heart failure (table 3). Patients in the HIV-positive group who died had low CD4 cell counts (mean 88 cells $/ \mathrm{mm}^{3}$ ), likely predisposing them to various infections, including VAP. They also had advanced HIV disease at the time of presentation. The most common diagnoses on admission among the HIV-positive group were non-PCP pneumonia, sepsis, and change in mental status, 
underlining the central role of infection as a main contributor to mortality in HIV-infected patients undergoing $\mathrm{MV}$ in the ICU.

The rate of weaning among the HIV-positive group was good for those who were admitted with non-HIV/non-AIDS related diseases, and actually was better than in the non-HIV group, though this difference did not achieve statistical significance. Previously reported outcomes for patients with HIV infection admitted to the ICU who did not have respiratory failure was better than for those who did..$^{3,10}$ Notably, prolonged ventilation (ie, tracheostomy) was more common in HIV-negative patients than HIV-infected patients in the present study. We defined patients who had a tracheostomy as having prolonged mechanical ventilation because they were sent to a ventilator step down unit or to a nursing home with a ventilator facility and continued on ventilators. Weaning in the HIV-negative patients required one month on average, with some patients never being weaned due to debility. Based on our observation, we found that patients in the HIV-positive group were more debilitated with decreased functional capacity, which increased their mortality compared to the HIV-negative group who survived the ICU stay following their severe illnesses, but needed tracheostomy for further management. The decreased mortality and increased need for long-term management in HIV-negative patients likely accounts for the higher rate of tracheostomy.

Univariate followed by multivariate analysis suggested elevated temperature, low blood pressure, low albumin, and VAP as the predictors of mortality (table 6). CD4 count was part of the analysis, but it was not a significant predictor of mortality; morbidity in terms of VAP was high as the CD4 count decreased. Nutritional status has been shown to be a very important factor in HIV-infected patients requiring ICU care in several studies. Albumin is one of the factors that is taken into consideration when assessing nutritional status. The data presented here suggest that low serum albumin is a predictor of mortality consistent with findings by De Palo et $\mathrm{al}^{3}{ }^{3}$ Wachter et $\mathrm{al},{ }^{15}$ and Powel et al ${ }^{1}$ which all showed that patients with higher serum albumin had better outcomes. Powell et al ${ }^{1}$ also demonstrated that decreased serum albumin, the need for MV, respiratory failure as an ICU admission diagnosis (compared to all other diagnoses), and PCP were all associated with decreased survival in HIV-infected patients.

Ventilator-associated pneumonia is an important cause of morbidity and mortality despite improved antimicrobial therapy, supportive care, and prevention, ${ }^{16}$ and it is a complication in approximately $28 \%$ of patients who receive MV. ${ }^{17}$ The incidence of VAP increases with the duration of MV. Estimated rates are 3\% per day for 5 days, $2 \%$ per day for days 6 to 10 days, and $1 \%$ after day $10 .{ }^{17}$ Although VAP is a common problem in intubated and ventilated patients, previous studies have not clearly demonstrated that VAP is associated with increased mortality or prolonged MV in HIVinfected patients. Fagon et $\mathrm{al}^{18}$ demonstrated that pneumonia occurring in ventilated patients, especially pneumonia due to Pseudomonas and Acinetobacter species, is associated with considerable mortality, in excess of that resulting from the underlying disease alone, and it significantly prolonged the length of stay in the ICU. Despite the fact that HIV is an epidemic and many HIV-positive patients are intubated, the 2005 American Thoracic Society/Infectious Diseases Society of America (ATS/IDSA) guidelines ${ }^{19}$ on the management of adults with hospital-acquired, ventilator-associated, and health care-associated pneumonia excluded immunocompromised patients, including HIV-infected patients. ${ }^{19}$ Since other studies have reported Pseudomonas aeruginosa and Staphylococcus aureus as the predominant causes of nosocomial bacterial pneumonia in the ICU in both HIV- and non-HIV-infected patients, ${ }^{20,21}$ management for both groups remains the same. In our population, we isolated Pseudomonas aeruginosa, Klebsiella pneumoniae, and Staphylococcus aureus as the most common causative organisms in the HIV-positive group, while the predominant organism isolated from the non-HIV group with VAP included Acinetobacter baumannii. Regardless, as far as the treatment and management of VAP in immunocompromised patients is concerned, there is no study or data which suggests they should be treated differently, so they are treated as per the ATS/IDSA guidelines. Our study suggests that patients with HIV infection are at increased risk for VAP, probably because they have very low immunity; the majority of the 39 patients that had VAP in the HIV-positive group had AIDS (table 4).

Our study had several limitations. While the number of subjects in the study group was small, this is the first study to include a control population. Additionally, we were not able to retrieve data on in-hospital mortality, average length of hospital stay, or to follow patient outcomes beyond ICU stay. In this study, the length of intubation was considered length of ICU stay, since patients were sent to other hospital units or institutions following extubation or tracheostomy. Furthermore, due to poor medication compliance rates and extremely poor health in many patients which limited communication, we had little information regarding patient HIV medications. Few HIV-infected patients were on HAART upon admission (8 of 55), and HAART was suspended in all patients upon admission to the ICU in order to prevent interference with treatment for acute illness. It is possible that the absence of HAART in patients with low CD4 counts may have had an effect on the mortality outcomes in this study. However, a 2009 study by Powell et $\mathrm{al}^{1}$ found that although the long-term benefits of anti-retroviral therapy are indisputable, there may not be any short-term benefit of antiretroviral therapy in ICU patients with a critical illness. The low rate of HAART use prior to admission is likely to have had an effect on the percentage of patients being admitted for AIDS-related issues and may therefore impact on mortality, affecting the generalizability of the study to populations in which HAART use is higher. Another limiting factor of our study is the especially high rate of VAP observed in both HIVpositive and HIV-negative patients $(71 \%$ and $25 \%$, 
respectively), which may have significant impact on the outcome of mortality and limit generalizability of these findings to patient populations with lower rates of VAP.

\section{Conclusions}

In comparison with HIV-negative patients requiring MV, HIV-infected patients requiring MV in our study experienced increased mortality and morbidity, including VAP, particularly if the patient had AIDS and was admitted secondary to an HIV-related illness. Low serum albumin, elevated temperature, low systolic blood pressure, and VAP were predictors of poor outcomes, including mortality, in our study. Since VAP is associated with a poor prognosis, discovering ways to reduce its incidence in the HIV-infected patient may improve outcome.

\section{Acknowledgements}

Dr. Pathak had full access to all data in the study and takes responsibility for the integrity of the data and accuracy of data analysis. Drs. Hurtado Rendon, Ciubotaru, Atrash, Prasad Rao Gagadam, Bhunia, Prasad Mallampalli, Vegesna, and Mani Dangal contributed to the study concept and design, analysis and interpretation of the data, drafting of the manuscript, and critical revision for important intellectual content. The work for this study was performed at St. Barnabas Hospital (Bronx, NY). No potential conflicts of interest exist on the part of any author with any companies/ organizations whose products or services may be discussed in the article. The authors thank the Marshfield Clinic Research Foundation's Office of Scientific Writing and Publication (Marshfield, WI) for assistance in the preparation of this manuscript.

\section{References}

1. Powell K, Davis JL, Morris AM, Chi A, Bensley MR, Huang L. Survival for patients with HIV admitted to the ICU continues to improve in the current era of combination antiretroviral therapy. Chest 2009;135:11-17.

2. Coquet C, Pavie J, Palmer P, Barbier F, Legriel S, Mayaux J, Molina JM, Schlemmer B, Azoulay E. Survival trends in critically ill HIV-infected patients in the highly active antiretroviral therapy era. Crit Care 2010;14: R107

3. De Palo V, Millstein B, Mayo P, Salzman SH, Rosen MJ. Outcome of intensive care in patients with HIV infection. Chest 1995;107:506-510.

4. Centers for Disease Control and Prevention. 1993 revised classification system for HIV infection and expanded surveillance case definition for AIDS among adolescents and adults. Morbid Mortal Weekly Rep 1993;41:1-19.

5. Wachter RM, Luce JM, Turner J, Volberding P, Hopewell PC. Intensive care of patients with the acquired immunodeficiency syndrome. Outcome and changing patterns of utilization. Am Rev Respir Dis 1986;134: 891-896.

6. Rosen MJ, Clayton K, Schneider RF, Fulkerson W, Rao AV, Stansell J, Kvale PA, Glassroth J, Reichman LB, Wallace JM, Hopewell PC. Intensive care of patients with HIV infection: utilization, critical illnesses, and outcomes. Pulmonary Complications of HIV Infection Study Group. Am J Respir Crit Care Med 1997;155:67-71.
7. Narasimhan M, Posner AJ, DePalo VA, Mayo PH, Rosen MJ. Intensive care in patients with HIV infection in the era of highly active antiretroviral therapy. Chest 2004; 125:1800-1804.

8. Afessa B, Green B. Clinical course, prognostic factors, and outcome prediction for HIV patients in the ICU. The PIP (Pulmonary complications, ICU support, and prognostic factors in hospitalized patients with HIV) study. Chest 2000;118:138-145.

9. Nickas G, Wachter RM. Outcomes of intensive care for patients with human immunodeficiency virus infection. Arch Intern Med 2000;160:541-547.

10. Morris A, Creasman J, Turner J, Luce JM, Wachter RM, Huang L. Intensive care of human immunodeficiency virus-infected patients during the era of highly active antiretroviral therapy. Am J Respir Crit Care Med 2002;166:262-267.

11. Rosen MJ, De Palo VA. Outcome of intensive care for patients with AIDS. Crit Care Clin 1993;9:107-114.

12. Rosenberg A, Seneff M, Atiyeh L, Wagner R, Bojanowski L, Zimmerman JE. The importance of bacterial sepsis in intensive care unit patients with acquired immunodeficiency syndrome: implications for future care in the age of increasing antiretroviral resistance. Crit Care Med 2001;29:548-556.

13. Alves C, Nicolás JM, Miró JM, Torres A, Agustì C, Gonzalez J, Raño A, Benito N, Moreno A, Garcìa F, Millá J, Gatell JM. Reappraisal of the aetiology and prognostic factors of severe acute respiratory failure in HIV patients. Eur Respir J 2001; 17:87-93

14. Valdez H, Chowdhry T, Asaad R, Woolley IJ, Davis T, Davidson R, Beinker N, Gripshover BM, Salata RA, McComsey G, Weissman SB, Lederman MM. Changing spectrum of mortality due to human immunodeficiency virus: analysis of 260 deaths during 1995-1999. Clin Infect Dis 2001;32:1487-1493.

15. Wachter RM, Russi MB, Bloch DA, Hopewell PC, Luce JM. Pneumocystis carinii pneumonia and respiratory failure in AIDS. Improved outcomes and increased use of intensive care units. Am Rev Respir Dis 1991;143:251-256.

16. Niederman MS. Guidelines for the management of respiratory infection: why do we need them, how should they be developed, and can they be useful? Curr Opin Pulm Med 1996;2:161-165.

17. Cook DJ, Walter SD, Cook RJ, Griffith LE, Guyatt GH, Leasa D, Jaeschke RZ, Brun-Buisson C. Incidence of and risk factors for ventilator-associated pneumonia in critically ill patients. Ann Intern Med 1998;129:433-440.

18. Fagon JY, Chastre J, Hance AJ, Montravers P, Novara A, Gibert C. Nosocomial pneumonia in ventilated patients: a cohort study evaluating attributable mortality and hospital stay. Am J Med 1993;94:281-288.

19. American Thoracic Society; Infectious Diseases Society of America. Guidelines for the Management of Adults with Hospital-acquired, Ventilator-associated, and Healthcareassociated Pneumonia. Am J Respir Crit Care Med 2005; 171:388-416.

20. Petrosillo N, Nicastri E, Viale P. Nosocomial pulmonary infections in HIV-positive patients. Curr Opin Pulm Med 2005;11:231-235.

21. Franzetti F, Grassini A, Piazza M, Degl'innocenti M, Bandera A, Gazzola L, Marchetti G, Gori A. Nosocomial bacterial pneumonia in HIV-infected patients: risk factors for adverse outcome and implications for rational empiric antibiotic therapy. Infection 2006;34:9-16. 


\section{Author Affiliations}

Vikas Pathak, MD*,;, ; Iliana Samara Hurtado Rendon, MD*;

Shebli Atrash, MD*; Vinay Prasad Rao Gagadam, MD*;

Kaushik Bhunia, MD*; Syam Prasad Mallampalli, MD*;

Vijay Vegesna, MD*; Mahesh Mani Dangal, MD*;

Ronald L. Ciubotaru, MD"

*Department of Internal Medicine, St. Barnabas Hospital, Bronx, NY, USA

Department of Internal Medicine/Hospitalist, Marshfield Clinic, Marshfield, WI, USA

*Department of Pulmonology/Intensive Care, St. Barnabas

Hospital, Bronx, NY, USA

${ }^{I}$ Current affiliation: Pulmonary Disease and Critical Care Medicine, University of North Carolina School of Medicine, 130 Mason Farm Rd, Chapel Hill, NC 27599 